山्山FFRANÇAISE

$\supset \mathrm{DE}$

$\simeq$ PÉDAGOGIE

\section{Revue française de pédagogie}

Recherches en éducation

154 | janvier-mars 2006

La construction des politiques d'éducation : de nouveaux rapports entre science et politique

\title{
Les inégalités de réussite en EPS entre filles et garçons : déterminisme biologique ou fabrication scolaire?
}

Difference in results in PE between girls and boys: biological determinism or made up by school?

Die Erfolgsungleichheiten im Sport zwischen Jungen und Mädchen: biologischer Determinismus oder schulisches Erzeugnis?

Las desigualdades de éxito entre chicas y chicos en EPS : ¿ determinismo biológico o fabricación escolar?

\section{Cécile Vigneron}

\section{(2) OpenEdition Journals}

Édition électronique

URL : http://journals.openedition.org/rfp/146

DOI : $10.4000 /$ rfp. 146

ISSN : 2105-2913

Éditeur

ENS Éditions

Édition imprimée

Date de publication : 1 mars 2006

Pagination : 11-124

ISBN : 2-7342-1039

ISSN : 0556-7807

\section{Référence électronique}

Cécile Vigneron, «Les inégalités de réussite en EPS entre filles et garçons : déterminisme biologique ou fabrication scolaire ? ", Revue française de pédagogie [En ligne], 154 | janvier-mars 2006, mis en ligne le 01 mars 2010, consulté le 30 avril 2019. URL : http://journals.openedition.org/rfp/146 ; DOI :

$10.4000 /$ rfp. 146 


\title{
Les inég a lités de réussite en EPS entre filles et garçons: déterminisme biologique ou fabric ation scolaire?
}

\author{
Cécile Vigneron
}

En EPS, l'observation des éc ats de résultats aux examens entre filles et garçons contraste avec les analyses réalisées dans les autres disciplines. Les résultats des filles en EPS sont faibles et systématiquement inférieurs à ceux des garçons. Parallèlement, se combinent aux effets de genre, de puissants effets sociaux qui surprennent parfois dans un Contexte d'évaluation et de réalisation de performances sportives inférées à la détention de qualités physiques. Si les travaux en sociologie de l'éduc aion montrent que l'EPS se fond dans le moule traditionnel de l'exCellence scolaire en en embrassant les normes et les valeurs, le travail présenté ici expose une approche complémentaire qui s'attache aux représentations et attentes des enseignants de cette discipline, susceptibles de produire chez leurs élèves des deux sexes des inégalités de réussite sans rapport aucun avec leurs ressources physiologiques.

Descripteurs (TEE) : Comportement de l'élève, différence de sexe, éducation physique, réussite scolaire, rôle de l'enseignant.

En éduc aion physique et sportive (EPS), les résulEtats observés aux épreuves du b ac cataéat depuis 1986 témoignent d'une meilleure réussite des garçons par rapport aux filles (Davisse, 1986). Ce Constat surprend, en référence aux brillants résultats des filles dans les autres disciplines, mais il est aussitôt tempéré par une justification biologique. Les différences physiologiques et morphologiques entre filles et garçons sont avancées pour interpréter les écarts de performance. D'emblée ce postulat mérite quelque attention et il s'agira de requestionner Cette évidence : la production de performances physiques et sportives renvoie-t-elle exclusivement à la dimension biologique de l'individu? Autrement dit, Ce qui est physique échappe-t-il forCément au déterminisme social?

L'évaluation en EPS s'est longtemps limitée à la prise de performances en référence à des barèmes et tables de cotation sexuées, mais aujourd'hui, la certification au baccalauréat s'attache aux compétences et connaissances des élèves, à leurs conduites motrices et aux procédures mises en $œ u v r e$ pour réussir. Soucieux d'équité, tiraillés par la question des inégalités de potentiel et de développement des adolescents, les enseignants d'EPS ont relativisé l'usage de leurs chronomètres et de leurs décam ètres. L'évaluation des seules performances 
révélait une injustice profonde par sa référence à des facteurs biologiques, physiologiques, génétiques inégalement répartis entre les individus. Néanmoins, de façon inattendue, les inégalités de réussite perdurent : quel que soit l'outil de mesure, l'infériorité des filles apparaît. Dès lors, se pose la question du choix des apprentissages proposés en amont de l'évaluation par les enseignants. La Culture physique et sportive divise les individus selon leur sexe, leur âge, leurs revenus, etc. et l'école dans son souhait de transmettre à tous des savoirs communs, pourrait Creuser sinon engendrer des éc ats entre filles et garÇons par un enseignement enté sur une transmission de techniques corporelles particulières et éloigné des valeurs des filles. Une observation des pratiques sportives, des savoirs et pouvoirs moteurs des filles construits au fil de la socialisation et de l'éduc aon familiale pourrait alors expliquer leurs résultats inférieurs. Les travaux issus de la sociologie témoignent effectivement d'un investissement différencié des jeunes selon leur sexe et leur origine sociale dans les pratiques Culturelles et sportives, objets d'enseignement en EPS, mais n'envisagent guère la manière avec laquelle ce constat est appréhendé par l'école. La reconnaissance et l'observation quotidienne de conduites motrices distinctes entre les sexes induisent sans doute aussi des attentes, des représentations chez les enseignants d'EPS, susceptibles d'influencer leurs pratiques pédagogiques.

L'objet de ce propos est donc d'examiner les éc ats de réussite en EPS $a u b$ ac cataéat, contradictoires avec les bons résultats des filles décrits par ailleurs, et passés sous silence dans les actuels travaux en sociologie de l'éduc aion. Il se propose de mener une étude CirConspecte des différences biologiques qui distingueraient filles et garçons au lycée et sont souvent présentées Comme suffisantes pour expliquer les éc ats de réussite en EPS. L'attention sera aussi portée sur les effets des facteurs sociaux qui pourraient disqualifier l'exclusivité du recours au facteur biologique comme facteur principal impliqué dans la fabric aion des inégalités. Enfin, il s'agira de s'interroger sur les méc aismes scolaires qui produisent ou transforment des différences physiques, Culturelles, en inégalités de réussite scolaire.

\section{LES PREMIÈRES OBSERV ATIONS SANS LENDEM AIN}

En France, Cest A. Davisse (1986) qui a initié les travaux relatifs aux éc ats de résultats entre filles et garçons en EPS. Pressentis mais jamais réellement mesurés, elle en dénonce l'ampleur, a ec MVolondat (1987), précisant les paramètres susceptibles de les éclairer : filières et activités évaluées (1). Ce travail pointe aussi des différences considérables entre genres au fil des séries du baccalauréat. G. Combaz (1992) approfondit Ces recherches. Il vérifie les effets de "séries " et note l'effet paradoxal de l'âge qui pénalise les filles quand il valorise les garçons. Son travail montre aussi que les élèves issus des catégories sociales défavorisées sont moins performants à l'examen avec des écarts entre filles et garçons qui sont d'autant plus grands que l'on se situe aux niveaux les plus "bas" de la hiérarchie sociale. P. Fontayne (1999) observe les écarts de réussite au Collège non plus selon le sexe, mais selon le genre des individus (2). Son étude s'appuie sur les théories de la motivation et il vérifie que les performances des élèves en EPS sont médiées par l'adhésion aux stéréotypes culturels et sensibles aux effets de genre. Enfin plus récemment, B. David (2000) dans une vaste étude menée à l'INRP dresse un bilan national des écarts. Les résultats affinent les travaux antérieurs en spécifiant notamment les différences selon les activités sportives évaluées (3).

Enfin, il faut rappeler aussi l'originalité des travaux de S. Coupey (1995) à l'école primaire, qui souligne les conséquences motrices sur les élèves, filles et garçons, des différences d'attente de la part des enseignants et de leurs choix d'objets d'apprentissage. Cette observation suggère que les écarts de performance entre les sexes, Certifiés au baccalauréat, peuvent résulter d'une sédimentation au fil $d u$ temps mais aussi d'une construction scolaire par des mécanismes scolaires diffus.

\section{LES FACTEURS INDIVIDUELS DE PERFORM ANCE EN EPS : BIOLOGIQUES OU SOCIAUX ?}

En EPS, Ia suprématie des garçons est associée à l'émergence et à l'utilisation de capacités physiques et physiologiques présumées supérieures. Une récente recherche relative aux facteurs de la performance motrice réalisée sur plus de 3500 adolescents (Narring et al., 1997) permet d'établir la part des Choses. Les mesures de la taille, du poids, du BMI (Body Mass Index), de la tension artérielle, du développement pubertaire ont été réalisées Conjointement à des passations de questionnaires et d'épreuves de condition physique. Cette étude conclut à une infériorité féminine sur le plan des dimensions et ressources physiques qui se double d'une implacable régression des aptitudes des filles dès la puberté. 
Synthétisant de multiples travaux antérieurs, D. Costill \& J. H. Willmore (1998), Confirment aussi l'infériorité des ressourCes physiques des femmes. Plus petites, disposant de moins de masse musCulaire mais de davantage de masse graisseuse, les femmes témoignent d'un potentiel physiologique inférieur à celui des hommes relativement aux systèmes cardio-pulmonaire, musculaire, endocrinien, etc.

Aujourd'hui pourtant, la communauté scientifique dans son ensemble modère ces constats: les recherches et analyses antérieures sont souvent fortement marquées par une idéologie qui prône le déterminisme biologique ou souffrent de carences méthodologiques (4). Les travaux plus récents (Vidal \& Benoit-Browaeys, 2005) attestent que le sexe serait un facteur de peu d'importance dans la prédiction ou la limitation des performances physiques. Ce seraient davantage les compétences acquises, l'entraînement, la motivation, Ia maîtrise de techniques efficaces qui distingueraient entre eux les individus. Les performances exceptionnelles d'alpinistes, de marathoniennes et de nageuses indiquent l'ampleur des possibilités physiologiques de femmes Convaincues et entraînées. La modicité des écarts entre les actuels records masculins et féminins Contraste avec les fossés qui séparent hommes et femmes ordinaires. Des expériences contradictoires (Hudson, 1994) avec des enfants invités à Iancer à bras cassé montrent que les écarts de distance Constatés initialement avec le bras préférentiel s'estompent lors que le jeune lance avec son bras inhabituel... Ces exemples invitent à penser que les ressources biologiques d'un individu n'épuisent qu'une infime part des déterminants de la perfor$m$ ance, quand les compétences acquises, l'entraînement, la maîtrise de techniques efficaces pèseraient Considérablement.

\section{LA SOCIALISATION FAMILIALE ET LES PRATIQUES SPORTIVES}

Le corps des enfants en effet, se modèle en premier lieu dans la famille et les expériences motrices, dans les attitudes exigées, dans les jeux encouragés ou au contraire interdits selon le sexe, qui s'avèrent déterminants pour la vie physique future des adultes. Les travaux relatifs aux processus de socialisation précoces (Belotti, 1974 ; Tap, 1985 ; Lloyd, 1994 ; Zimmermann \& Reavil, 1999) Confirment que l'on encourage assez peu les filles à s'activer, à explorer l'espace afin de ménager leur "fragilité " quand on sollicite les garçons à s'engager dès leur plus jeune âge dans une motricité diversifiée.

Mauss (1966) montre ainsi que les usages du Corps entre hommes et femmes se distinguent partiCulièrement dans tous les domaines quotidiens. Chaque groupe social se distingue par des usages particuliers, eux-mêmes spécifiques à chacun des sexes. Pour Bourdieu (1977), "le Corps dans Ce qu'il a de plus naturel en apparence, C'est-à-dire dans les dimensions de sa conformation visible (volume, taille, poids, etc.) est un produit social, la distribution inégale entre les classes des propriétés corporelles s'accomplissant à travers différentes médiations telles que les conditions de travail et les habitudes en matière de consommation ". La société apprend à chacun des sexes, les gestes qui lui Conviennent et les techniques sportives heurtent souvent les usages traditionnels du corps des femmes.

Parallèlement, les pratiques Culturelles et sportives des adolescents distinguent sensiblement filles et garçons. Une étude de R. Boyer (1991), montre que filles et garçons des différentes classes sociales ne partagent pas la même Culture, ni les mêmes loisirs, notamment sportifs. Concernant l'accès des femmes aux pratiques sportives, C. Louveau (2002) explique aussi qu'au delà des propos optimistes relatifs à la fém inisation et à la massification des pratiques sportives, il existe des exclues du sport. Femmes de milieux populaires ou ruraux, lycéennes des établissements professionnels, femmes et filles issues de l'immigration n'accèdent $p$ as aux pratiques sportives sous le poids de contraintes à la fois financières, matérielles, familiales et Culturelles.

Même si les jeunes de quinze à vingt-quatre ans constituent la catégorie de français la plus sportive (Bouillet \& Truchot, 2002 ), les adolescentes se détachent progressivement des pratiques sportives au fil de leur scolarité (Davisse \& Louveau, 1991). Elles sont moins souvent licenciées ou engagées dans des pratiques compétitives que leurs frères. Enfin, les jeunes filles concentrent leurs pratiques sportives autour de peu d'activités largement étiquetées féminines: gymnastique, danse et patinage. Si le sport est associé au masculin pour nombre de Chercheurs (Matteo, 1986 ; Ignico, 1989 ; Harry, 1995) Chaque activité est aussi perçue comme sexuellement marquée (Czisma, Wittig \& Schurr, 1988; Marin, 1988 ; Pelett \& Harrison, 1988 ; Salminen, 1990 ; Koivula, 1995) et Cette différenciation éclaire des 
investissements variables selon les sexes. Les enfants apprennent très tôt, dans leur famille et à l'école, que certaines pratiques sont conformes à leur sexe; ils tentent alors de se conformer au modèle de genre qui leur est attribué. Ainsi, les scores décevants des filles en EPS au b ac cataéat peuvent être observés non plus comme la Conséquence exclusive et regrettable de transformations biologiques pubertaires mais plutôt comme un aboutissement de leur activité modérée, de leur inappétence pour Certains sports. Plus encore, une possible implic aion de l'institution scolaire elle-même dans la fabric aion de ces différences en EPS entre filles et garçons émerge. En reconnaissant et en valorisant Certaines pratiques et activités éloignées des champs Culturels quotidiens des filles, elle contribue sans doute à ac coître les éc ats.

\section{D'AUTRES MÉCANISMES EN JEU : LA FABRICATION SCOLAIRE DES DIFFÉRENCES}

Pour P. Perrenoud (1984), l'école dicte, sélectionne et Choisit de transmettre Certains Contenus, elle laisse l'appropriation aléatoire d'autres savoirs aux hasards de la socialisation familiale, asociative, ou médiatique. De nombreux travaux ont montré que les programmes scolaires ne sont pas l'aboutissement d'une progressive sédimentation des savoirs mais résultent davantage d'un processus de sélection et de discrimination parmi les Connaissances disponibles (Forquin, 1990). Effectivement, @ processus aboutit pour M. Duru-Bellat (1994) et N. Mosconi (1995) à la Constitution de Curricula scolaires largement empreints des normes masCulines. Le "masculin neutre "s'impose à tous au sein de l'école et finalement, sous couvert d'enseignement commun des enfants des deux sexes, le champ culturel masculin est constitué et imposé Comme référence universelle. Mais étudiant les scolarités des filles, elles décrivent aussi d'autres méc a nismes en jeu dans la classe, comme les interactions, les formes de groupements, les effets d'attente, etc., qui limitent selon elles les filles dans la pleine expression de leurs potentialités.

Les travaux de R. Rosenthal et L. Jacobson (1975) repris par J. E. Brophy et T. L. Good (1986) déCrivent préCisément Ces effets d'attente chez les enseignants. Pour chacun de ses élèves, le maître attend un comportement et des performances. En Conséquence, il adopte lui même une conduite qui indique discrètement à l'élève les résultats et aspirations prévus à son égard, l'affecte, modifie sa perception de lui même et l'invite à se conformer aux espérances du professeur. B. Zazzo (1982), R Sirota (1988), M Duru-Bellat (1994), C. Baudoux et A. Noircent (1995), A. Durand-Delvigne (1995), N. Mosconi (1995) et C. Zaidmann (1995) explorent les processus et les effets des perCeptions et des représentations des enseignants en classes mixtes. Elles montrent Comment les garÇons sont Considérés Comme des sous-réalisateurs, appréciés pour leur dynamisme et leur singularité, et destinataires d'interactions nombreuses et variées. Les filles renvoient une image positive à l'enseignant de son travail : appliquées, persévérantes, discrètes, elles reçoivent cependant moins d'interactions et Celles-Ci valorisent davantage la présentation que les résultats. Au final, une discrète prédilection entoure les garçons: félicités pour leurs résultats mais davantage tancés pour leur désinvolture, ils renforcent leur estime d'eux-mêmes quand, implicitement, les filles associent leurs réussites à l'intensité de leur travail et doutent de leurs compétences. Les réussites des filles paraissent d'ailleurs, encore aujourd'hui, bien moins avérées, éphémères et partielles, sans doute menacées et fragilisées par des mécanismes diffus tant sociaux que scolaires.

\section{LA RÉUSSITE EN EPS : QUELS ÉLÈVES ?}

Les résultats présentés ici sont issus d'une recherche Conduite en 2001 sur deux populations associées d'enseignants d'EPS et d'élèves d'une de leurs classes de terminale dans l'académie de Reims. Un état des lieux a été établi à partir d'un questionnaire élève et d'un recueil pour chaque lycéen de ses résultats en EPS pour les trois activités physiques sportives ou artistiques (APSA) sujettes à l'épreuve du baccalauréat. Ces données associées aux variables individuelles (5) des élèves ont été ensuite croisées, avec les résultats d'un questionnaire "professeur" adressé aux enseignants d'EPS de ces mêmes candidats bacheliers (6), visant à saisir leurs représentations et leurs perceptions des élèves et des APSA.

Trois questions sous-tendaient le travail:

En quoi et Comment les facteurs biologiques ontils réellement ou non un lien avec la réussite des élèves en EPS? Les pratiques sociales, et tout particulièrement sportives, ont-elles un effet sur les notes en EPS des élèves au baccalauréat? Enfin,

114 Revue française de pédagogie, n¹54, janvier-février-mars 2006 
Tableau I. - Note moyenne obtenue en EPS au baccalauréat selon le sexe des élèves

\begin{tabular}{|l|c|c|c|c|c|}
\hline Sexe de l'élève & Moyenne & $\mathrm{N}$ & Écart type & Minimum & Maximum \\
\hline Fille & 13.25 & 753 & 2.01 & 5 & 19 \\
\hline Garçon & 14.46 & 492 & 2.10 & 5 & 20 \\
\hline Total & 13.73 & 1245 & 2.13 & 5 \\
\hline
\end{tabular}

dans quelle mesure les représentations et effets d'attentes des enseignants Conjugués avec les choix de Contenus pèsent-ils également sur les réussites différenciées dans cette discipline entre filles et garçons?

La population étudiée est Constituée de 753 filles et 492 garçons et les résultats collectés pour Cette session du b ac calaéat 2001 confirment les recherches antérieures: la note moyenne obtenue en EPS $a u b$ ac cauréat $p a r$ les filles s'établit à $13,25 / 20$ quand Celles des garçons est de $14,46 / 20^{* \star *}(7)$.

L'étude des notes obtenues selon le sexe et l'âge montre que les lycéennes en retard de deux années ou plus réussissent moins bien que leurs $c$ anarades à l'heure $(12,97$ contra 13,29*) quand les élèves en avance surpassent le groupe $\left(13,41\right.$ Contra $\left.13,05^{* *}\right)$. On observe l'effet inverse chez les garçons (8).

Un relevé des données biométriques des élèves a été effectué pour observer les relations éventuelles entre stature et résultats obtenus en EPS. Soumettre tous les élèves de l'échantillon à des tests d'aptitude physique s'avérait irréaliste, aussi les observations se sont limitées au poids et la taille. On a arbitrairement Considéré Comme grands les garçons mesurant plus de $1,80 \mathrm{~m}$ ou grandes les filles de plus de $1,70 \mathrm{~m}$, et Comme petits les garÇons mesurant moins de $1,70 \mathrm{~m}$ ou petites les filles mesurant moins de 1,60 $\mathrm{m}$. Les mensurations observées distinguent les élèves selon les séries et les $C$ aégories sociales d'origine. Les filles des séries scientifiques sont plus grandes $(37,1 \%$ d'entre elles mesurent plus de $\left.1,70 \mathrm{~m}^{* * *}\right)$ et plus lourdes aussi que leurs $C$ an arades Ce qui ne se vérifie pas pour les garçons. De même, les filles de $c$ ares sont elles aussi plus grandes mais plus minces que les autres lycéennes. Cependant, les résultats obtenus en EPS ne sont pas significativement différents selon Ce Critère, notamment Chez les garçons: seules les filles petites ou lourdes obtiennent des notes moyennes significativement inférieures à leurs cam arades. (12,97 pour les petites par exemple contre $13,31^{* *}$ aux candidates plus grandes ou encore 12,55 contra $13,33^{* *}$ pour les lyCéennes de plus de $65 \mathrm{~kg}$ ).

Une attention croisée entre sexe, origine sociale et note d'EPS révèle un écart de 0,41 point ${ }^{*}$ au détriment des enfants d'ouvriers comparativement aux autres lycéens quand les enfants de cadres bénéfiCient eux de 0,31 point supplémentaire ( $\mathrm{ns}$ ). Les filles sont plus affectées (0,36 point en moins) Comparativement aux autres filles que les garçons $(0,21$ point en moins). Les fils de cadres ne tirent pas avantage de leur origine quand leurs sœurs obtiennent 0,48 point supplémentaire par rapport aux autres candidates.

Enfin, les pratiques Culturelles et sportives distinguent nettement les élèves : filles et garçons, ne s'investissent pas à la même hauteur dans les pratiques sportives au gré de leurs origines sociales, de leurs séries au baccalauréat. Ils ne choisissent pas non plus les mêmes activités, les mêmes modalités ou structures de pratique, y consacrent plus ou moins de temps et y associent des objectifs fort distincts allant du simple loisir à la haute compétition. Si globalement sept garçons sur dix, quelles que soient leur série de baccalauréat, déclarent une pratique sportive régulière, seules les filles de série $S$ affichent une telle assiduité. Les filles de séries technologiques s'engagent par exemple deux fois moins que les garçons des mêmes classes, le plus souvent hors institution et majoritairement dans des buts d'entretien ou de loisir.

Une pratique sportive régulière apporte aux filles 1,12 points ${ }^{* *}$ supplémentaires à leur note d'EPS au baccalauréat quand cette même caractéristique apporte 1,35 points ${ }^{* *}$ aux garçons (9). La pratique en Club, encadrée et compétitive, semble davantage liée avec un meilleur niveau de réussite en EPS à l'examen (cf. tableau II). Le temps consacré à la pratique et le niveau atteint sont aussi largement Corrélés à Ia note obtenue (10). L'atteinte d'un niveau départemental permet d'emblée aux filles de se distinguer 
quand il reste insuffisant pour les garçons. L'ac ession à un niveau de compétition régional puis national va de pair, à chaque pallier suc ossif avec une hausse de deux points sur la note finale par exemple.

Le choix des différentes pratiques sportives, lui même associé aux origines sociales des élèves n'est pas sans Conséquence sur les résultats obtenus. Si la pratique de Certains sports, comme les sports Collectifs ou le tennis va de pair avec de meilleurs résultats, inversement Celle de la danse conduit à une b ase sensible des résultats observés. Plus encore, le footb 由, oc ope une place à part au sein des sports collectifs, ses adeptes se voyant plutôt attribuer des notes $b$ ases. Ces constats laissent penser qu'au-delà de Compétences strictement spécifiques aux différents sports, il existe sans doute des savoirs et savoirs-être plus transversaux et plus diffus, reconnus, valorisés ou rejetés par l'institution, que Certains élèves se seraient appropriés par le biais de la socialisation.

Tableau II. - La note obtenue en EPS au b ac catréat selon les variables individuelles des élèves

\begin{tabular}{|c|c|c|c|c|c|c|}
\hline \multirow[b]{2}{*}{ Variables explicatives } & \multicolumn{3}{|c|}{ Filles } & \multicolumn{3}{|c|}{ Garçons } \\
\hline & Coefficient $\beta$ & T de Student & Signification & Coefficient $\beta$ & T de Student & Signification \\
\hline Constante & 13,182 & 65,132 & 0,000 & 13,865 & 61,001 & 0,000 \\
\hline Pratique sportive de l'élève & 0,462 & 2,545 & $0,011^{*}$ & 0,547 & 2,167 & $0,031^{*}$ \\
\hline Pratique compétitive & 1,171 & 4,774 & $0,000^{* * *}$ & 0,749 & 3,219 & $0,001^{* *}$ \\
\hline $\begin{array}{l}\text { Mesure moins de } 1,60 \mathrm{~m} \text { (filles) } \\
\text { ou } 1,70 \mathrm{~m} \text { (garçons) }\end{array}$ & $-0,244$ & $-1,429$ & 0,153 (ns) & 0,157 & $-0,595$ & $0,552(\mathrm{~ns})$ \\
\hline Pèse plus de 65 kg (filles) & $-0,649$ & $-2,632$ & $0,009^{* *}$ & & & \\
\hline Est "à l'heure ou en avance" & 0,317 & 2,090 & 0,037 & $-0,201$ & $-1,068$ & 0,286 (ns) \\
\hline Est scolarisé(e) en série littéraire & $-0,805$ & $-3,796$ & $0,000^{* * *}$ & $-0,071$ & $-0,146$ & $0,884(\mathrm{~ns})$ \\
\hline $\begin{array}{l}\text { Est scolarisé(e) en série } \\
\text { économique et sociale }\end{array}$ & $-0,470$ & $-2,376$ & $0,018^{*}$ & $-0,102$ & $-0,382$ & $0,703(\mathrm{~ns})$ \\
\hline $\begin{array}{l}\text { Est scolarisé(e) en séries } \\
\text { technologiques }\end{array}$ & $-0,838$ & $-4,243$ & $0,000^{* * *}$ & $-0,565$ & $-2,581$ & $0,010^{*}$ \\
\hline CSP du Chef de famille = ouvrier & $-0,235$ & $-1,397$ & $0,163(\mathrm{~ns})$ & $-0,361$ & $-1,536$ & $0,125(\mathrm{~ns})$ \\
\hline Pratique un sport Collectif & 0,308 & 0,985 & $0,325(\mathrm{~ns})$ & 0,668 & 2,110 & $0,035^{*}$ \\
\hline Pratique la danse & $-0,213$ & $-0,777$ & $0,438(\mathrm{~ns})$ & & & \\
\hline Pratique le football & & & & $-0,229$ & $-0,726$ & $0,468(\mathrm{~ns})$ \\
\hline Joue au tennis & 0,539 & 1,482 & $0,139(\mathrm{~ns})$ & 0,219 & 0,593 & $0,552(\mathrm{~ns})$ \\
\hline Est inscrite à I'UNSS & 0,469 & 3,298 & $0,001^{* *}$ & & & \\
\hline
\end{tabular}

NB : Uhe analyse multivariée (1 1permet de démêler l'impact net d'une variable explicative sur la variable dépendante, iCi la note obtenue en EPS au $b$ acçuréat, les autres variables étant maintenues constantes.

Il Convient de lire ainsi: Les filles obtiennent au b accæuréat une note moyenne de 13,182 le fait de pratiquer par ailleurs un sport accroit la note de 0,462 point Comparativement à une fille présentant exactement les mêmes $c$ eactéristiques individuelles. La Colonne $T$ de Student permet de mesurer la part d'explication de chaque variable. Si $t>2,6$, la variable est très explicative, entre 1,9 et 2,6 son pouvoir explicatif est moindre, entre 1,6 et 1,9, la variable apporte peu d'explication, en deçà, son pouvoir explic af n'est pas avéré. La colonne Signification indique la fiabilité du résultat. Des valeurs $p<0,05$ indiquent moins de 5\% de risque d'erreur $\left({ }^{*}\right) ; p<0,01$ : moins de $1 \%$ de risque d'erreur $\left(^{* *}\right)$ et lorsque $p<0,001$, les risques d'erreur sont extrêmement faibles. 
Ce tableau tend à placer l'EPS aux côtés des autres disciplines en pointant les effets identiques de variables comme les CSP, les pratiques Culturelles, etc. Il révèle néanmoins des variables remarquables : taille et poids, âge, pratiques sportives, etc., dont les effets sont parfois Contradictoires selon le sexe des élèves ou seulement significatifs pour les filles. Les résultats, croissants pour les garçons et décroissants pour les filles, avec l'âge reflètent sans doute les effets de la socialisation qui invite les jeunes filles à se restreindre dans leur motricité pour aspirer à davantage de séduction quand l'expression de l'identité $m$ asculine passe à cet âge par la puissance ou la force. Le poids de Certaines variables initialement sous-estimées comme la pratique à l'association sportive du lycée ou la nature des sports pratiqués laisse par ailleurs envisager d'autres liens possibles qui se tisseraient dans la relation pédagogique ou dans les choix de Contenus enseignés. À Cette étape de la recherche, il est apparu pertinent d'explorer les mécanismes scolaires susceptibles d'intervenir dans la construction d'une note d'EPS au baccalauréat.

Deux directions principales sont présentées ici parmi les pistes explorées. L'une renvoie aux choix des enseignants en matière de Contenus enseignés, l'autre s'attache aux représentations et attentes des enseignants de Cette discipline. Les professeurs responsables des classes interrogées ont été invités à caractériser particulièrement les filles et les garçons qui réussissent, ou au contraire échouent, dans leur discipline. Ils ont listé les APSA sensibles de Ce point de vue, C'est-à-dire Celles qui Creusent ou réduisent les écarts entre les deux sexes. Par ailleurs, ils ont été sollicités pour prédire les notes de leurs élèves selon leur sexe en distinguant les plus faibles et les meilleurs (12).
Les explications des échecs des filles portent invariablement sur une modestie présumée de leurs ressourCes physiques. Les enseignants estiment que les filles ont des qualités physiques restreintes, un vécu sportif limité, qu'elles ne maîtrisent pas les techniques sportives et n'apprécient pas la compétition. Ils insistent sur les piètres aptitudes dont elles disposeraient qui expliqueraient ainsi leurs perfor$m$ ances. Inversement, ils interprètent les difficultés de quelques garçons exclusivement par un manque de travail, d'assiduité ou de sérieux, qualités par ailleurs toujours mises en avant chez les filles qui restent volontaires, appliquées, sérieuses. L'idée de garÇons sous-réalis ateurs est ici reprise, avec la certitude pour eux d'un fort potentiel insuffisamment mobilisé par manque d'effort ou d'application contrairement aux filles. Cette comparaison accentue le faible niveau estimé des ressources des filles.

Les activités où les garçons excellent sont selon eux l'athlétisme, le football et le volley-ball, plus généralement les sports collectifs et d'opposition. Les filles seraient expertes en danse, gymnastique et natation parfois aussi en athlétisme. Les seules activités selon les enseignants, où filles et garçons peuvent espérer rivaliser renvoient aux activités d'excellence des filles (danse exclue) - natation, gymnastique, badminton, athlétisme - quand Celles où l'écart paraît maximal reprennent le palmarès des garçons. Les enseignants justifient leurs propositions en décrivant des activités plus propices aux filles pour leur absence d'opposition et la part limitée de l'engagement physique nécessaire; Ce qui leur permet aussi d'entrer en comparaison avec les garçons dans quelques activités, ce sont les effets de l'entraînement et de l'exercice ou l'application de barèmes différenciés. Ils légitiment leurs affirmations relatives aux activités les plus discriminantes pour

Tableau III. - Résultats obtenus dans les différentes activités physiques et sportives par les filles et les garçons en EPS au b ac carréat

\begin{tabular}{|c|c|c|c|c|c|c|c|c|c|c|}
\hline APSA & $\begin{array}{l}\text { Sports } \\
\text { Collectifs }\end{array}$ & $\begin{array}{l}\text { Bad- } \\
\text { minton }\end{array}$ & $\begin{array}{c}\text { Tennis } \\
\text { de Table }\end{array}$ & Raquettes & $\begin{array}{c}\text { Gymnas - } \\
\text { tique }\end{array}$ & $\begin{array}{l}\text { Athlé- } \\
\text { tisme }\end{array}$ & Natation & Escalade & Danse & Plein air \\
\hline Filles & 11,83 & 12,13 & 12,33 & 12,20 & 13,37 & 12,17 & 13,95 & 12,50 & 13,27 & 12,98 \\
\hline$\%$ de filles notées & $69,5 \%$ & $47,1 \%$ & $29,7 \%$ & $76,9 \%$ & $26 \%$ & $55,4 \%$ & $24,4 \%$ & $8,4 \%$ & $11,3 \%$ & $15,3 \%$ \\
\hline Garçons & 14,03 & 13,94 & 14,31 & 14,08 & 13,46 & 13,35 & 13,90 & 12,29 & 12,50 & 13,26 \\
\hline$\%$ de garçons notés & $88,8 \%$ & $54,5 \%$ & $29,5 \%$ & $83,9 \%$ & $13 \%$ & $57,5 \%$ & $27 \%$ & $10,2 \%$ & $1,8 \%$ & $16,5 \%$ \\
\hline Différence & 2,2 & 1,81 & 1,98 & 1,88 & 0,09 & 1,18 & $-0,05$ & $-0,21$ & $-0,77$ & 0,28 \\
\hline
\end{tabular}


les filles par la mobilisation incontournable de ressources énergétiques, par une obligation de coopération ou encore par la mise en œuvre de choix tactiques élaborés.

L'étude des épreuves soumises à l'évaluation vient alors interroger les discours des enseignants. Au sein de leurs projets pédagogiques observés, les professeurs d'EPS organisent leur enseignement en privilégiant largement Certaines activités comme les sports Collectifs $(77,1 \%$ d'élèves évalués en sports Collectifs), les jeux de raquettes $(79,6 \%$ d'élèves évalués) ou l'athlétisme (56,2\% d'élèves évalués), en réduisant la danse $(7,6 \%$ d'élèves évalués) ou la gymnastique $(20,9 \%$ d'élèves évalués) à la portion congrue. Bien que les notes obtenues par les filles dans les activités de raquettes comme le b aminton soient très inférieures à celles des garçons, ils les Considèrent néanmoins comme neutres (13) en terme de genre.

Ce tableau permet de Constater que les activités sollicitant fortement les ressources physiques ne s'avèrent pas les plus pénalisantes pour les filles. Les écarts maximaux sont observés en volley-ball et badminton, activités par ailleurs très rarement pratiquées par les élèves hors de l'institution scolaire et qui recueillent largement l'adhésion des filles. Par ailleurs, l'analyse des unités d'enseignement associant trois activités pour constituer l'épreuve finale du baccalauréat montre que les combinaisons retenues par les enseignants s'appuient principalement sur les activités les plus préjudiciables aux filles en terme de note, ce qui accentue les écarts constatés.

Les enseignants ont été aussi invités à prévoir les notes qu'ils estimaient probables pour leurs élèves à l'issue de l'ensemble des épreuves. Dans Cette perspective, les enseignants (14) ont prévu quasiment un point supplémentaire au meilleur élève garçon ou à l'élève garçon médian du groupe comparativement aux mêmes élèves filles. Pour eux, seuls les lycéens les plus faibles obtiendraient une note équivalente quand un point séparerait les excellences masCulines et féminines.

Globalement, les notes obtenues au final par les élèves sont légèrement supérieures aux prévisions des enseignants. Ces derniers envisagent systématiquement des résultats moindres pour les filles à positionnement équivalent et la réalité confirme voire accentue les attentes: les notes finales des garçons dépassent leurs prévisions. La médiocrité des plus faibles est exacerbée dans les pronostics mais la réalité infirme les pronostics. Les enseignants annoncent des notes inférieures à celles qu'ils attribueront en définitive et se montrent au final plus généreux pour les garçons qu'ils favorisaient pourtant déjà largement dans leurs prévisions.

Interrogés de la même manière sur les résultats et notes qu'ils s'attendent à obtenir, les élèves s'avèrent modestes et luCides. Ils restent en dessous des valeurs effectives, les filles affichant une tendance plus pessimiste. Elles espèrent en moyenne 12,5 et obtiendront au final 13,25. Les garçons attendent en moyenne 13,87 et ils valideront Collectivement 14,46. De façon inattendue, les élèves se trompent moins que les enseignants.

\begin{tabular}{|c|c|c|}
\hline $\begin{array}{c}\Delta: \text { note prévue/ } \\
\text { obtenue pour les élèves }\end{array}$ & Filles & Garçons \\
\hline $\begin{array}{c}\Delta: \text { note prévue/ } \\
\text { obtenue pour les professeurs }\end{array}$ & $-0,75$ & $-0,59$ \\
\hline
\end{tabular}

Tableau IV. - Résultats prévus par les enseignants puis obtenus pour les élèves filles et garçons en EPS au b ac camréat.

\begin{tabular}{|l|c|c|c|c|c|c|c|c|c|}
\hline Note & $\begin{array}{c}\text { Garçon le } \\
\text { plus faible } \\
\text { du groupe }\end{array}$ & $\begin{array}{c}\text { Garçon } \\
\text { moyen }\end{array}$ & $\begin{array}{c}\text { Meilleur } \\
\text { élève } \\
\text { garçon }\end{array}$ & $\begin{array}{c}\text { Fille la } \\
\text { plus faible } \\
\text { du groupe }\end{array}$ & $\begin{array}{c}\text { Fille } \\
\text { moyenne }\end{array}$ & $\begin{array}{c}\text { Meilleure } \\
\text { élève } \\
\text { fille }\end{array}$ & $\begin{array}{c}\text { Écart } \\
\text { constaté } \\
\text { G/F les } \\
\text { plus faibles }\end{array}$ & $\begin{array}{c}\text { Écart } \\
\text { constaté } \\
\text { G/F } \\
\mathrm{m} \text { oyens }\end{array}$ & $\begin{array}{c}\text { Écart } \\
\text { Constaté } \\
\mathrm{m} \text { eilleurs } \\
\mathrm{G} / \mathrm{F}\end{array}$ \\
\hline Prévue & 9,37 & 13,14 & 17,34 & 9 & 12,32 & 16,33 & $+0,37$ & $+0,82$ & $+1,01$ \\
\hline Obtenue & 11,7 & 14,46 & 16,81 & 10,2 & 13,25 & 16,17 & $+1,5$ & $+1,21$ & $+0,64$ \\
\hline Différence & $+2,33$ & $+1,32$ & $-0,53$ & $+1,2$ & $+0,93$ & $-0,16$ & & \\
\hline
\end{tabular}

118 Revue française de pédagogie, $n^{\circ} 154$, janvier-février-mars 2006 
Majoritairement, les enseignants interrogés estiment qu'il est facile d'avoir une bonne note en EPS mais ils réfutent la proposition qu'il puisse être plus facile pour une fille d'avoir une bonne note en EPS que pour un garçon (pour 64,1\% d'entre eux). Seuls $3,1 \%$ des enseignants appuient Cette opinion quand $18,8 \%$ d'entre eux la soutiennent pour les garçons. Enfin, 42,2\% d'entre eux Considèrent l'importance des qualités physiques dans l'attribution d'une bonne note en EPS.

Ces derniers résultats suggèrent que les choix pédagogiques des enseignants d'EPS traduisent une intériorisation de certains modèles et stéréotypes Culturels, justement révélée par les inégalités de réussite entre filles et garçons. Les effets d'attentes ou les représentations des enseignants d'EPS vis-à-vis de leurs élèves, des APSA, influencent sans doute leur enseignement, et altèrent peut-être le sentiment de compétence des filles, produisant alors des inégalités légitimées par des alibis physiologiques ou Culturels, qui obèrent une remise en cause fondamentale de leurs pratiques pédagogiques.

\section{UNE CONSTRUCTION SOCIALE ET SCOLAIRE}

Justification première et suffisante, la possession de ressources physiques comme présupposé à la réussite en EPS vacille ici quelque peu. Rien ne permet en effet d'affirmer dans cette étude qu'on réussit mieux en EPS lorsque l'on est grand, svelte ou, au contraire, trapu. Ce qui apparaît, C'est bien davantage le croisement d'un faisceau de variables, beaucoup plus sociales et scolaires que biologiques, déterminantes pour la réussite dans cette discipline.

Les filles des séries technologiques par exemple attestent de résultats médiocres $(12,68$ Contra $\left.13,44^{\star * *}\right)$. Elles sont issues à $65,3 \%$ de milieux ouvriers, employés, retraités, Chômeurs (Contre $39,1 \%$ des filles des séries scientifiques); seules 43,2\% d'entre elles pratiquent une activité sportive $(58,7 \%$ des candidates au baccalauréat de série $S)$ et de surcroît sont souvent de taille moyenne sinon petite $(33,3 \%$ d'entre elles mesurent moins de $1,60 \mathrm{~m}$ Contre $16,3 \%$ des filles de série $S)$ et/ou en surcharge pondérale. Il Convient alors de démêler la part du social et du biologique. C. Pociello (1999) rappelle que la hiérarchie des tailles suit et reproduit précisément la hiérarchie des classes sociales. Assez caricaturalement les grands minces se rencontrent parmi les étudiants, professions libérales et cadres supérieurs et les petits gros chez les artisans, agriculteurs... Les facteurs alimentaires, les conditions de vie, les valeurs Culturelles associées à l'image du corps constituent un ensemble d'éléments tout à fait déterminants et capables de modifier sensiblement les aspects et aptitudes physiques des individus. II faut donc prendre quelque recul avec une logique imprudente fondée sur la génétique ou la biologie pour identifier les facteurs sociaux susceptibles d'éclairer ces constats (15).

La réussite en EPS des filles scolarisées dans les séries scientifiques et majoritairement issues des classes sociales élevées illustre encore ce propos. Grandes, impliquées dans des pratiques d'activités sportives distinctes de celles de leurs camarades, Ces lyCéennes rencontrent aussi les attentes de l'école. Pour C. Louveau (2002) ou A. Baudrit (1994) les sports investis par les femmes et les jeunes sont surdéterminés par la position sociale. Les filles des classes supérieures et moyennes se distinguent en affichant dans leurs loisirs et pratiques sportives des préférences pour des activités moins Congruentes avec leur sexe. R. Boyer (1991) déCrit une unité masculine des loisirs des garçons de toutes les classes sociales autour des mêmes pratiques sportives ou de la micro-informatique alors qu'elle souligne une multiplicité féminine en pointant les choix singuliers des filles des classes supérieures davantage en rupture avec les stéréotypes sexués relatifs aux pratiques sportives. Pour elle, "Chaque classe traite ses filles différemment et les filles de chaque classe construisent leurs propres réponses à leur situation; se maintiennent donc les rôles féminins les plus traditionnels chez les filles des classes populaires, alors qu'apparaissent des pratiques à connotation masculine chez les filles des classes supérieures... ».

Cette étude renvoie alors aux choix des savoirs enseignés en EPS. Ceux-Ci reposent largement sur les activités sportives institutionnalisées, Compétitives, Considérées comme partie intégrante de la Culture et donc dignes d'être enseignées. Pourtant, pour G. Combaz (1992) ou P. Arnaud (1996) Cet enseignement sis sur des techniques sportives insCrites dans l'histoire du sport masculin ne véhicule pas les mêmes significations pour tous les élèves quelles que soient leurs origines sociales, Culturelles et plus encore sexuelles. Ces savoirs ne Constituent pas nécessairement une Culture universelle, ayant valeur incontestable au regard de critères de légitimité scientifique ou d'excellence artistique mais 
reflètent sans doute davantage d'autres déb es idéologiques. Une sensible évolution semble néanmoins poindre avec l'introduction dans les programmations de nouvelles activités en apparence plus féminines. Mais à bien y regarder, derrière l'es $c$ æade, le b aminton ou le cirque, se dessinent les contours d'une motricité toujours empreinte de force, de vitesse, de puissance, de prise de risque, valeurs éminemment masculines sur lesquelles se grefferont ultérieurement Contenus et évaluations. P. Arnaud montre que si les mêmes sports sont enseignés aux filles et aux garçons, Cest parce qu'ils s'appuient sur des techniques "iso-sexuées", abitrairement choisies selon une logique de performance et de normalisation masculines fort éloignées des techniques du Corps féminines décrites par l'anthropologie. P. Liotard (1996) souligne l'illusion d'une éduc aion physique féminine. Pour lui, historiquement, derrière l'éduc a tion d'un élève asexué, s'organise en fait une éduC aion physique pensée et produite par et pour les hommes, Cest-à-dire posée comme universelle qui adapte ses méthodes et ambitions à la fragilité des filles. En EPS, le modèle corporel et Culturel masCulin prévaut.

Les résultats présentés soulignent aussi le poids des représentations des enseignants d'EPS. Pour M. Gilly (1980), les objectifs éduc afs spécifiques des enseignants influencent les perceptions de ces derniers. Le contexte dans lequel l'élève est appréhendé modifie les représentations des adultes et établit une sorte de c are de valeurs qui b Asent et orientent la perception sociale. Ce qui prend sens aux yeux des enseignants, Ce sont les attitudes perÇues dans les situations les plus en adéquation avec le projet éducatif institutionnel poursuivi. En EPS, les enseignants élaborent leurs représentations à partir d'un registre spécifique de situations motrices, Compétitives et énergétiques qui s'avèrent être significatives pour eux et répondent en conséquence aux attentes et objectifs institutionnels qui légitiment leur fonction et discipline. Ces représentations des enseignants d'EPS se construisent sur la base d'un prélèvement et d'une hiérarchisation d'indices partiCuliers, sélectionnés justement pour inférer de Comportements caractéristiques sans doute assez loin des conduites des filles. Les enseignants d'EPS sont sensibles à un Certain dynamisme, à un esprit compétitif qui les renvoie à leur propre image sportive passée et à leur identité professionnelle. Implicitement, ils estiment les élèves qui par leurs pratiques sociales ou sportives s'approchent des Choix de l'école: Compétiteurs, adhérents de clubs, pratiquants de disciplines Collectives, ou olympiques. Ils restent aussi admiratifs et peut-être nostalgiques devant les performances des jeunes sportifs quand les piètres réalisations des filles irritent particulièrement les enseignantes en référence à leurs propres performances passées. Enfin, ils reconnaissent les élèves sérieux, appliqués, investis par exemple à l'association sportive du lycée parce qu'ils leur renvoient une image positive de leur travail et de leur dis Cipline.

Ces représentations déterminent sans doute aussi les interactions effectuées en EPS. M. Durand (2001) illustre abondamment les représentations négatives et l'ensemble des stéréotypes de sexe avec lesquels les professeurs d'EPS dépeignent les filles puis interagissent avec elles : les filles «n'aiment pas le sport", "elles sont assises", elles "discutent". II multiplie les exemples d'interactions à leur égard qui pointent le peu d'ambition pour elles, le registre essentiellement affectif des encouragements et Conseils prodigués quand il souligne le caractère technique et spécifique des feedb ak adressés aux garçons. S. Siedentop (1983) souligne les préjugés et les injustices à l'égard des filles, il interpelle les enseignants d'EPS sur les clichés rapides associés aux concepts de masculinité et féminité. Son travail révèle une large dis Crimination à l'égard des filles qui s'illustre dans les interactions et allusions souvent sexistes, dans l'attribution de rôles subalternes, dans les modalités de constitution des groupes, dans les humiliations publiques, dans la qualité du matériel distribué, etc. II insiste sur la modestie des exigences fixées aux filles, sur la médiocrité des feed $b$ ak reçus qui contredit la précision et les exigences des messages transmis aux garçons. Cette analyse rejoint les travaux de $S$. Coupey qui a démontré qu'en EPS, "les garçons progressent davantage que les filles dans les classes des maîtres qui disent avoir des exigences différentes pour les filles et les garçons".

Pour terminer, D. Trouilloud et P. Sarrazin (2002) réfutent pour I'EPS la réalité d'effets d'attente. Ils postulent qu'en EPS, les enseignants disposent de Compétences leur permettant de prédire, sans influencer, les performances des élèves à partir d'une observation rigoureuse des prestations des élèves et d'une analyse précise et pertinente de leurs ressources fondée sur des indicateurs fiables Comme les résultats antérieurs. Ils pourraient repérer les élèves motivés, autonomes, ou ceux qui montrent des aptitudes initiales conséquentes. Notre étude Contredit quelque peu leurs propos même si, finalement, ils Conviennent que d'autres attentes 
plus subjectives - comme la couleur de peau, la tenue vestimentaire, l'attractivité physique ou le sexe des élèves - peuvent parasiter l'appréciation portée.

L'enjeu de l'égalité des chances à l'école, Cest Celui de neutraliser les inégalités issues du patrimoine social. Faut-il écrire biologique et social pour l'EPS? L'objet de cet article a été de montrer Comment la Construction d'une note en EPS $a u b$ ac C æauréat s'enracine dans un enchevêtrement de ressources physiques, psychologiques, Culturelles et de mobiles d'agir ou de résister des élèves, filles et garçons. Mais il pointe aussi les choix particuliers de l'école et de ses enseignants, l'aspect androcentré des Contenus enseignés, déterminés à partir d'activités sportives, masculines et Compétitrices ou les effets d'attente et les représentations des professeurs d'EPS ou encore les conséquences de leur propre rapport au savoir sportif transmis, élément essentiel de leur identité.

Les résultats présentés invitent à revenir sur deux types d'enjeux de nature différente : enjeux scolaires, incontestablement, mais aussi et plus largement, enjeux politiques, idéologiques.

La pérennisation depuis vingt ans de tels éc ats entre filles et $g$ arçons sans réactions fortes de l'institution interroge. Elle souligne sans doute la place minime réservée à l'EPS dans le système scolaire, discipline de peu de poids dans les cursus, les examens et les choix d'orientation. Si l'EPS dérange quelque peu dans l'ordre scolaire établi en bous Culant les hiérarchies et les normes d'exCellence, elle les conforte finalement aussi: à la fois en reconnaissant aux garçons la domination physique, sportive, valeur éminemment masculine mais aussi en permettant l'ac es d'une minorité socialement déterminée de filles à Cette excellence. Certes, l'institution par de sensibles pressions, ou des procédures arithmétiques tente d'infléchir le phénomène, d'égaliser les performances. Mais au-delà de cette pure dimension scolaire se pose la question de l'éducation physique des filles et de la conception du rôle social qui leur est assigné et dont le corps en est une expression directe.

Pour C. Dowling ( $\left.\begin{array}{lll}2 & 0 & 10\end{array}\right)$ les féministes ont obtenu des avancées incontestables sur les pôles économiques, intellectuels, juridiques mais elles ont minimisé les enjeux du pouvoir physique. Or pour elle, C'est en réalité Celui qui leur permettra d'accéder pleinement à l'égalité. Avec F. Héritier (1996) elle montre que le contrôle des femmes, dans toutes les sociétés passe par la domination et l'assujettissement de leur corps, elle décrit les maints procédés $m$ is en œuvre par les grands appareils idéologiques, religion, éducation, santé, dans toutes les sociétés pour veiller à garder le contrôle des forces des femmes, et leur réserver, sinon leur assigner, Certains gestes et usages du Corps. Elle s'interroge sur les raisons qui incitent à limiter la motricité des filles, à ne pas leur apprendre à courir, sauter, Iancer, etc. Dans ce débat, I'EPS peut alors apparaître Comme émancipatrice en cherchant justement à développer les Compétences physiques des filles. Pourtant, notre étude montre que par le choix de ses Contenus et méthodes, par les attentes et représentations de ses acteurs, elle contribue, elle aussi, à renforcer la faiblesse apprise des filles. Comparées aux garçons, confrontées à des activités masculines, leurs erreurs ou échecs considérés avec bonhomie et mansuétude, leurs ressources mises en doute et minimisées par leurs enseignants, les filles se persuadent progressivement de leur médiocrité physique, en admettent le caractère inéluctable sinon naturel et investissent d'autres domaines. Les considérer exclusivement en victimes passives serait Cependant une erreur. Face à l'étendue du combat à entreprendre pour rivaliser sur le plan physique avec les garÇons, les filles effectuent sans doute des choix plus subtils et pertinents; lucides sur les attentes qu'on entretient à leur égard dans la société de demain et sur les rôles sociaux qui leur sont réservés, elles savent aussi qu'elles ont sans doute plus à perdre qu'à gagner à briller par leurs exploits sportifs scolaires et gravissent progressivement mais méthodiquement les échelons.

Se pose alors la question des moyens d'une EPS réellement ambitieuse, égalitaire et émancipatrice pour les filles et non pas seulement reproductrice. Si I'EPS ne peut prétendre à transformer les rôles sociaux, les stéréotypes sexués de toute une société, il faudra sans doute se défaire d'une indifférence bienveillante sur les difficultés des filles. Constater les médiocres réalisations des filles en EPS, $m$ ais ne pas prendre en compte les différences Culturelles et sociales entre individus sous Couvert d'égalité de traitement entre les élèves, C'est peutêtre assumer la responsabilité de maintenir délibérément des biais sexistes dans cette discipline.

Cécile Vigneron alvigne@club.fr

Lycée Robert-Doisneau (Vaulx-en-Velin)

Centre de recherche et d'innovation sur le sport (CRIS) Université Claude-Bernard - Lyon 1 
(1) Elle constate que les notes des filles sont inférieures à celles des garçons de 0,5 point en moyenne avec des éc ats pouvant monter à 2 points dans Certaines activités ou séries. Elle observe que 1,46 points peuvent différencier des filles issues de séries scientifiques et technologiques.

(2) À la notion de genre, sont reliés des Comportements, atitudes, activités, rôles et représentations de soi marqués so Cialement et Culturellement par une empreinte sexuée quand le sexe est généralement seulement associé aux c aactéristiques biologiques qui différencient hommes et femmes. L'étude de Fontayne s'appuie sur une version simplifiée du BSRI de S. Bem (1986) questionnaire standardisé d'auto-description qui détermine un profil et un indice d'androgynie par comparaison et $m$ ise en rapport de scores surdes échelles de masculinité et de fém inité.

(3) Les trois activités les plus souvent évaluées: ahlétisme, volley$b$ a et $b$ aminton sont les plus discriminantes. Elles rassem blent à elles seules, selon J.-P. Cleuziou, 53,5\% des pratiques évaluées en terminale. En sports de raquettes, 1,87 points séparent filles et garçons, 1,76 en sports Collectifs et 1,39 en athlétisme quand les activités artistiques - qui ne concernent que $2,9 \%$ des garçons et $9,5 \%$ des filles - permettent aux ly Céennes de devancer très légèrement leurs cam arades.

(4) Les études considérées ont été réalisées sur des populations d'individus ordinaires, en l'absence de contrôle de variables Comme l'âge, l'entraînement - plus fréquent Chez les hommes ou l'éducation. Les performances des champions ou seulement de sujets masculins et féminins entraînés à l'identique contredisent les résultats observés. Les comparaisons entre populations masculines et féminines se satisfont d'un examen des moyennes alors que des Chevauchements importants de résultats indiquent que de nombreuses femmes réalisent des scores équivalents aux hommes quand de nombreux hommes validen des scores inférieurs à bien des femmes. De nombreux tests sont réalisés en l'absence de standardisation, notamment pour prendre en compte les facteurs taille et poids. Les perfor$\mathrm{m}$ ances sont recueillies lors d'expérimentations fortement $m$ ar quées socialement et Culturellement comme le lancer à bras cassé qui révèle un apprentissage et une technique plutôt m as Culins.

(5) Outre les variables sociologiques habituelles, les élèves ont par exemple répondu à des questions relatives à leurs pratiques sportives extra-scolaires, leurs loisirs, leurs Cursus scolaires, leurs motivations, etc., et ont aussi renseigné plusieurs items Concernant leurs parents et les pratiques sportives et Culturelles familiales présentes ou passées.

(6) L'échantillon était constitué de soixante-quatre enseignants pour lequel les variables sexe, âge, formation et qualification, type d'établissement ont été contrôlées. Les résultats des élèves Concernent 1245 candidats à l'examen.

(7) La significativité de ce résultat a été mesurée à partir d'un test de $\chi^{2}$. Par Convention, les seuils de significativité sont sym bolisés du moins au plus significatif: ${ }^{*}: p<0,05 ;{ }^{* *}: p<0,01$ ${ }^{* \star *}: p<0,001$ (ns) signalant un effet non significatif. Ici $p=0,000$.

(8) Les garçons présentant deux ans ou davantage de retard, obtiennent une moyenne de 14,56/20 en EPS pour 14,44/20 aux ly Céens nés à partir de 1982

(9) En réalité, les garçons non sportifs sont extrêmement rares et constituent sans doute une population un peu atypique.
(10) Chaque heure de pratique hebdomadaire supplémentaire apporte 0,22 point aux filles et 0,25 point aux garçons.

(11) Les modèles d'analyse en régression multivariée prennent la forme de fonction suivante: $Y=a_{0} x_{0}+a_{1} x_{1}+a_{2} x_{2}+\ldots a_{n} x_{n}$. $Y$ représente la variable dépendante, ici la note finale EPS au baccalauréat estimée à 13,182 dans le modèle présenté pour les filles et 13,865 pour les garçons. $x_{0}, x_{1} \ldots x_{n}$ sont les variables indépendantes ou explicatives; dans le tableau ici présenté, Ce sont la pratique d'une activité sportive, la taille, le poids, la série, Ia CSP du chef de famille, la pratique d'un sport Collectif, l'âge, etc. Les coefficients $a_{0}, a_{1} \ldots a_{n}$ (dans le modèle présenté, repérés par la lettre $B$, Coefficient non standardisé donnent une mesure de l'effet des variables $x_{0}, x_{1} \ldots x_{n}$. La significativité de Cet effet est appréciée par un test de Student (Colonne t).

(12) Chaque enseignant était invité à placer sur une échelle de 0 à 20 "la note prévue pour la moins bonne fille du groupe, la moyenne des filles, la note prévue pour la meilleure fille du groupe". La même question Concernait les garçons. Ces prévisions ont été comparées par une méthode de tri à plat (mini, maxi, médiane, moyenne, écart-type) avec les notes réellement posées par chaque enseignant dans son groupe à ces six élèves caractéristiques.

(13) Le football, le rugby, les sports de combat (boxe, lutte et judo) et plus généralement les sports Collectifs sont, parmi les activités qu'ils enseignent, Celles que les professeurs d'EPS estiment les plus masculines. Concernant les activités plutôt féminines, la danse, la gymnastique et la gymnastique rythmique et sportive (GRS) sont largement énoncées aux côtés de quelques activités très discrètes comme l'aérobic, ou la natation synchronisée et l'acrogym. Le badminton serait pour les enseignants le sport neutre par excellence aux côtés du volley-ball puis de la natation.

(14) En moyenne, les enseignants hommes et femmes réalisent des projections assez similaires : les professeurs hommes se montrent plus négatifs vis-à-vis des filles, ils prévoient pour elles des notes moyennes sensiblement plus faibles que leurs collègues femmes et Ceci pour les filles médianes ou performantes au sein du groupe. À l'inverse, ils se montrent plus optimistes vis-à-vis des garçons que leurs collègues femmes en projetant pour eux entre 0,20 et 0,73 point en moyenne de plus.

(15) Selon l'INSEE, la hiérarchie de la taille reproduit celle de la société. Les hommes cadres dominent de leur taille $(175,6 \mathrm{~cm})$ toutes les autres CSP dans une hiérarchie parfaitement ordonnée où les ouvriers occupent le dernier échelon $(171,9 \mathrm{~cm})$. $28 \%$ des hommes cadres supérieurs dépassent $180 \mathrm{~cm}$ Contre $14 \%$ des agriculteurs ou $17 \%$ des ouvriers. Chez les femmes, le même constat est réalisé même si les écarts de taille entre CSP restent plus minimes: Une femme cadre mesure en moyenne 162,8 cm pour 160,8 cm Chez les ouvrières. Concernant le poids, la tendance s'inverse. L'écart est plus grand entre une femme cadre $(56,9 \mathrm{~kg})$ et une ouvrière $(62,5 \mathrm{~kg})$ qu'entre deux hommes issus des mêmes CSP $(71,3 \mathrm{~kg}$ pour un cadre et $72,8 \mathrm{~kg}$ pour un ouvrier).

Le phénomène d'obésité tou che aussi aujourd'hui environ $10 \%$ de femmes en France et $6 \%$ d'hommes. Ce sont les ouvrières qui présentent le rapport poids/taille le plus élevé. Les principales explications avancées sont des phénomènes héréditaires $\mathrm{m}$ ais surtout une alimentation déséquilibrée et un manque évident d'exercice physique. 
ARnaud P. (1996). "Le genre ou le sexe? Sport féminin et Changement social (XIX $-X X^{e}$ siècle) ». In P. Arnaud \& T. Terret (éd.), Histoire du sport féminin : tome $2:$ sport $m$ asculin-sport féminin : éducation et société. Paris. L'Harm attan, p. 147-183.

Baudoux C. \& Noircent A. (1995). "Culture mixte des classes et stratégies des filles". Revue française de pédagogie, n०110, p. 5-15.

BAUDRIT A. (1994). "Les pratiques sportives des enfants". Revue STAPS, n० 35, p. 19-75.

Belotti E. G. (1974). Du Côté des petites filles. Paris : Éd des femmes.

BEM S. L. (1986). "Au-delà de l'androgynie : quelques préCeptes osés pour une identité de sexe libérée". In M.-C. Hurtig \& M.-F. Pichevin (éd.), La différence des sexes, questions de psychologie. Paris : Éd. Tierce, p. 251-270.

Bouillet C. \& TRuchot G. "Les jeunes". In P. Mignon \& G. Truchot (Coord.), Les pratiques sportives en France: enquête 2000. Paris : Ministère des Sports : Institut national du sport et de l'éducation physique, p. 35-40.

BouRdieu P. (1977). "Rem arques provisoires sur la perception sociale du corps". Actes de la recherche en science sociales, $\mathrm{n}^{\circ} 14$, p. 51-54.

Boyer R. (1991). "Identité masculine, identité féminine parmi les lycéens". Revue française de pédagogie, n०94, p. 13-18

BRophy J. E. \& Good T. L. (1986). "Teacher Behaviour and Student Achievement". In M. C. Wittrock (éd.), Handbook of Research on Teaching. New York: Macmillan, p. $328-375\left[3^{\mathrm{e}}\right.$ éd.]

Cleuziou J.-P. (2000). "L'analyse des menus et des notes". In B. David (dir.), Éducation physique et sportive : la Certification au baccalauréat. Paris: INRP, p. 77-124.

Combaz G. (1992). Sociologie de l'Éducation physique. Paris: PUF.

Costill D. L. \& Willmore J. H. (1998). Physiologie du sport et de l'exercice physique. Bruxelles: De Boeck.

Coupey S. (1995). "Pratiques d'éducation physique et sportive au CP et différences de performance entre filles et garçons". Revue française de pédagogie, $n^{\circ} 110$, p. $37-49$

Czisma K. A; Wittig A. F. \& SchurR K. T. (1988). "Sports stereotypes and gender". Journal of Sport and Exercise Psychology, vol. 10, p. 62-74.

DAVID B. [dir.] (2000). Éducation physique et sportive : la certification au baccalauréat. Paris: INRP.

DAVISSE A. (1986). "Les mixités en EPS». Revue EPS, $n^{\circ} 197$, p. 56-57.

DAVISSE A. (1999). "EPS : réussite des filles et mixité ". In F. Vouillot (éd.), Filles et garçons à l'école : une égalité à construire. Paris: CNDP, p. 65-73.

Davisse A. \& Louveau C. (1991). Sports, école, société : la part des femmes : féminin, $m$ as Culin et activités physiques et sportives. Joinville-le-Pont [Val-de-Marne] : Actio.
Davisse A. \& Louveau C. (1998). Sports, école, société : la différence des sexes. Paris : L'Harmattan.

Davisse A. \& Volondat M. (1987). "Mixité : pédagogie des différences et didactiques". Revue EPS, n²06, p. $53-56$.

Dowling C. (2002). Le mythe de la fragilité. Paris: Éd. du Jour.

DuRAnd M. (2001). Chronomètre et survêtement: reflets de l'expérience quotidienne d'enseignants en éducation physique. Paris : Revue EPS.

DuRAnd-Delvigne A. (1995). "Jeu du soi et du genre : les effets structurels de la co-éducation". Les cahiers du MAGE, $\mathrm{n}^{\circ} 1$, p. 9-16.

DuRu-Bellat M. (1994-1995). "Filles et garçons à l'école, approches sociologiques et psycho-sociales". Revue française de pédagogie, $n^{\circ} 109, p .111-141$ \& n०110, p. $75-109$.

Fontayne P. (1999). Motivation et activités physiques et sportives: influence du sexe et du genre sur la pratique du sport et de l'éducation physique. Thèse de doctorat: sciences biologiques fondamentales et appliquées: psychologie, université Paris 11 .

ForQuIN J.-C. (1990). École et Culture. Bruxelles: De Boeck.

GILLY M. (1980). Maître-élève : rôles institutionnels et représentations. Paris : PUF.

Good T. ; Sikes J. \& Brophy J. (1973). "Effects of Teacher Sex and Student sex on Classroom Interaction". Journal of Educational PsyChology vol. 71, p. 74-87.

HaRRY J. (1995). "Sport ideology, attitudes tow ard women, and anti-homosexual attitudes". Sex Roles, vol. 32, p. $109-116$.

HéRITIER F. (1996). Masculin, fém in in : la pensée de la différence. Paris : O. Jacob.

Hudson J. (1994). "It's mostly a Matter of Metric". In D. M. Costa \& S. R. Guthrie (éd.), Women and Sport. Champaign: Hum an Kinetics Books, p. 143-162.

IGNico A. A. (1989). "Development and verification of gender-role stereotyping index for physical activities". Perceptual and Motor Skills, vol. 68, p. 1067-1075.

KoIvUla N. (1995). "Ratings of gender appropriateness of sports participation: Effects of gender-based schematic processing". Sex Roles, vol. 33, n7-8, p. 543-557.

LIOTARD P. (1996). "L'impossible spécificité de l'éducation physique féminine". In P. Arnaud \& T. Terret (éd.), Histoire du sport féminin: tome 2 : sport masculin, sport féminin : éducation et société. Paris : L'Harmattan, p. $185-211$.

LLOYD B. (1994). "Différences entre sexes". In S. MosCovici (dir.), PsyChologie sociale des relations à autrui. Paris : Nath an, p. 280-299.

LOUVEAU C. (2002). "Enquêter sur les pratiques sportives des français : tendances lourdes et problèmes méthodologiques". In P. Mignon \& G. Truchot (Coord.), Les pratiques sportives en France : enquête 2000. Paris : Ministère des Sports : Institut national du sport et de l'éducation physique, p. 135-154. 
MARIN M. (1988). "Gender-differences in sport and movement in Finland". International Review for Sociology of Sport, vol. 23, n०4, p. 345-359.

MATTEO S. (1986). "The effect of sex and gender schematic processing on sport participation". Sex Roles, vol. 15, p. 417-432.

Mauss M. (1966). Sociologie et Anthropologie. Paris : PUF [ $1^{\text {re }}$ éd. 1950$]$.

Mosconi N. (1995). "Division sexuelle des savoirs et Constitution du rapport au savoir". In M. de Manassein (éd.), De l'ég alité des sexes. Paris: CNDP, p. 204-218.

Narring F. ; Berthoud A. ; Cauderay M. ; Favre M. \& Michaud P. A. (1997). Condition physique et pratiques sportives des jeunes dans le canton de Vaud. Lausanne: Institut universitaire de médecine sociale et préventive : SEEPS

Pellett T. L. \& Harrison J. M. (1992). "Children's perceptions of the gender appropriateness of physical activities : A further analysis". Play and Culture, vol. 5, p. $305-313$.

Perrenoud P. (1984). La fabrication de l'excellence scolaire. Genève : Droz

Peterson P. L. \& Fennema E. (1985). "Effective teaching, student engagement in classroom activities, and sex-related differences in learning mathematics". American Educational Research Journal, vol. 22, $n^{\circ} 3$, p. $309-335$.

Pociello C. (1999). Sociologie du sport. Paris : Vigot.
Rosenthal R. \& Jacobson L. (1975). Pygmalion à l'école. Tournai [Belgique] : Casterman.

SALMINEN S. (1990). "Sex role and participation in traditionally inappropriate sports". Perceptual and Motor Skills, vol. 71, n 3, p. 1216-1218.

SiedentoP D. (1983). Developing Teaching Skills in Physical Education. Palo Alto : Mayfield [2 ${ }^{\mathrm{e}}$ éd.]; trad. et adapt. par M. Tousignant, P. Boudreau \& A. Fortier: Apprendre à enseigner l'éducation physique. Montréal : G. Morin, 1991.

Sirota R. (1988). L'école prim aire au quotidien. Paris: PUF.

TAP P. (1985). Masculin et fém in in Chez l'enfant. Toulouse : Privat

Trouilloud D. \& SARrazin P. (2002). "L'effet Pygmalion existe-t-il en éducation physique et sportive ? Influence des attentes des enseignants sur la motivation et la performance des élèves ". Science et motricité, $n^{\circ} 46$, p. 69-94.

Vidal C. \& Benoit-Browaeys D. (2005). Cerveau, sexe \& pouvoir. Paris: Belin.

ZaIDMANN C. (1995). "École, mixité, politiques de la différence des sexes". In M. de Manassein (Coord.). De l'égalité des sexes. Paris: CNDP, p. 219-232.

ZAZZO B. (1982). "Les Conduites adaptatives en milieu scolaire : intérêt de la comparaison entre les garçons et les filles ". Enfance, n4 , p. 267-282.

Zimmerman J. \& ReAVIL G. (1999). Raising our Athletes Daughters. New York: Doubleday. 